\title{
Histological and Immunohistochemical Evaluation for the Effect of Pilocarpine and Quercetin on Gamma-irradiated Parotid Salivary Glands
}

\author{
Said Mahmud Hani( ${ }^{(1)}$, AbdElnasserAbdElmawla ${ }^{(1)}$, Salwa Farid Ahmed ${ }^{(2) \#}$ and \\ Ashraf Mohamad Ali Emran ${ }^{(1)}$ \\ (1) Oral Biology Department, Faculty of Dental Medicine for Boys, Al-Azhar \\ University, Cairo, Egypt; (2) Health Radiation Research Department, National Centre \\ for Radiation Research and Technology, Atomic Energy Authority, Cairo, Egypt.
}

\begin{abstract}
$\mathbf{R}$ ADIOTHERAPY of patients with head and neck tumors usually causes damage to the salivary glands since these are most frequently included in the field of irradiation. This study aims at investigating the possible protective effect of pilocarpine or quercetin against radiation induced parotid gland damage. Forty-five adult male rats were divided randomly into three main groups as follows: Radiation group where the rats were exposed to a single whole body 6Gy $\gamma$-irradiation; pilocarpine- and quercetin-radiation groups where the rats injected intrapretoneally by a single dose of pilocarpine $(0.2 \mathrm{mg} / \mathrm{kg})$ or quercetin $(1.25 \mathrm{~g} / \mathrm{kg})$, respectively, 30 min later exposed to a single whole body $6 \mathrm{~Gy} \gamma$-irradiation. The parotid glands were stained with hematoxylin and eosin and immunohistochemical reaction for vascular endothelial growth factor A (VEGF-A). Irradiated glands revealed massive acinar atrophy, degeneration and cytoplasmic vacuolization while, the ducts showed cytoplasmic vacuolizations with loss of regular cell architecture. Pilocarpine or quercetin treatment before radiation exposure offered some protection effect manifested as reduced acinaratrophy, degeneration and vacuolization. The ducts showed mild cellular vacuolizations. Regarding VEGF-A immunoreactivity, gamma irradiated parotid gland showed intense expression and significantly higher area that has persisted up to sixteen weeks. Pilocarpine or quercetin treatment was associated with lesser degree and significantly lower area percent of VEGF-A expressionas compared to the radiation group. Pilocarpine or quercetin has some improvement for the injurious effects of radiation on salivary glands so, they can be used as radioprotective agents before radiotherapy schedules to decrease radiation side effects on salivary glands.
\end{abstract}

Keywords: Gamma-irradiation, Pilocarpine, Quercetin, Parotid, Vascular endothelial growth factor.

\section{Introduction}

During the last decades, there has been a substantial improvement in the treatment of head and neck cancer and radiotherapy is currently a widely used and important part of this treatment (El-Faramawy et al., 2013). In comparison with the surgical procedures, it shows better results because it can be used as a curative, adjuvant, neoadjuvant and palliative type of treatment and is often used in the conservative approaches, with protocols that preserve the organs and tissues (Marta et al., 2014). The major oral surrounding tissues that suffer from side effects when interacting with ionizing radiation from radiotherapy are the salivary glands. These tissues usually receive secondary doses from those delivered to the head and neck tumors, and although they have a low mitotic rate, salivary glands are considered to be extremely radiosensitive (Garg \& Malo, 1997). While the radiation damage to salivary glands is well known in the clinic by its sides effects, it is not known exactly what mechanism of destruction the ionizing radiation have on salivary glands. It is known that the serous acini are more radiosensitive than mucous acini (Coppes et al., 2002). Existing protection strategies comprise strict dental and oral hygiene, parotid-sparing radiation technique, and pharmacotherapy such as salivary substitutes and sialogogues (Chambers et al., 2004).

"Corresponding author email: salwaa_fared@yahoo.com 
Pilocarpine is a naturally occurring compound derived from the South American shrub, the pilocarpus jaborandi. This alkaloid plant is a cholinergic parasympathomimetic agent that acts as muscarinic agonist with a mild beta-adrenergic activity in addition to its ability to induce smooth muscle contraction and exocrine glands (Ferguson, 1993). Many studies demonstrated the efficacy of pilocarpine for the treatment of xerostomia caused by Sjogren's syndrome, radiotherapy or drug treatment. Pilocarpine is well tolerated and not only ameliorate patient quality of life, but it also inhibits the complications likewise (Vivino, 2001; Chitapanarux et al., 2008 and Looström et al., 2010).

Quercetin, a unique bioflavonoid, is found in fruits, vegetables, grains, bark roots, stem, flowers, tea and others (Crozier et al., 2009). It is considered a powerful antioxidant flavonoids against reactive oxygen species, produced during the normal oxygen metabolism or are induced by the exogenous damage (De Groot, 1994). In addition, it possesses anti-inflammatory (Comalada et al., 2005), vasodilatory (Nishida \& Satoh, 2009) and angiogenic effects (Sumi et al., 2013). Quercetin administration, not only improves the radiation-induced impaired salivary secretion, but it may also be an efficient way to maintain healthy salivary secretion (Takahashi et al., 2015). So, the purpose of this study is to investigate the efficiency of pilocarpine and quercetin as protector agents against radiation induced parotid glands damage.

\section{Experimental}

Forty-five adult male Sprague- Dawley rats weighing $150-250 \mathrm{~g}$ were used in this study. The experiment was performed after accommodation period in the laboratory environment for 7 days. The rats were housed in a room with controlled temperature $\left(25^{\circ} \mathrm{C} \pm 2{ }^{\circ} \mathrm{C}\right)$, humidity $(50 \% \pm 5 \%)$, about $12 \mathrm{~h}$ light/dark cycle and were fed on chew and water ad libitum. The experimental protocol followed the rules and regulations of the animal experimental studies approved by Ethical Committee of Faculty of Dental Medicine for Boys, AL-Azhar University, including their facilities diet and method of scarification.

The whole body irradiation of animals was performed at the National Center for Radiation Research and Technology (NCRRT), Cairo, Egypt, using Gamma cell 40. All animals were exposed to a dose of 6Gy $(137 \mathrm{Cs})$ at dose rate of $0.43 \mathrm{~Gy} / \mathrm{min}$.

The animals were divided randomly into three main groups $(\mathrm{n}=15)$. Radiation group $(\mathrm{R})$ wherethe rats were exposed to a single whole body $6 \mathrm{~Gy}$ $\gamma$-irradiation. Pilocarpine-radiation group (PR) and Quercetin-radiation group (QR) where the rats were injected intraperiotonially with a single dose of pilocarpine $(0.2 \mathrm{mg} / \mathrm{kg}$ ) (Sigma Chemical Co., St. Louis, MO), quercetin $(1.25 \mathrm{~g} / \mathrm{kg}$ ) (Sigma Chemical Co., St. Louis, MO) dissolved in $1 \mathrm{ml}$ of normal saline, $30 \mathrm{~min}$ later they were exposed to a single whole body $6 \mathrm{~Gy} \gamma$-irradiation.

At 1, 4 and 16 weeks after radiation exposure, 5 rats from each group were euthanasially decapitated. Then, the parotid glands were carefully dissected out and immediately fixed in neutral calciformol solution for $72 \mathrm{~h}$. The salivary glands were embedded in paraffin wax and paraffinized tissue sections were cut at 5-7 $\mu \mathrm{m}$ thick and prepared for hematoxylin and eosin stainfor histological evaluation and immunohistochemical reaction using the avidin-biotin technique for demonstration of the Vascular Endothelial Growth Factor A (VEGF-A) (Okada et al., 1999) were used to detect the vessels changes. The immunostained sections were digitized using a Zeiss Mirax automated slide scanner with an objective of $\times 20$ magnification. The VEGF-A expression was evaluated in 5 fields/slide, three slides/group using a score corresponding to the area percent of the positive cells. The digital image analysis was performed by importing the Mirax files into the image analyzer software Visiopharm integrater system (VIS).

The data were tabulated, coded then analyzed using the computer program SPSS (Statistical package for social science) version 17.0 for analysis of the immune expression of VEGF. The measured values were expressed as mean values \pm standard deviation. The statistical importance of the difference in these values between the different groups was estimated usingone- way analysis of variance (ANOVA). A $(\mathrm{P})$ value less than 0.05 was considered significant.

\section{Results}

\section{Histological examination}

One week post-irradiation

Gamma-irradiated parotid glands expressed many destructive changes including a massive loss of acinar architecture, sever acinar atrophy, 
vacuolization and degeneration. From the cellular point of view, the acini showed nuclear pleomorphism and hyperchromatism. The destructive manifestations of the ducts included loss of cellular architecture, cell vacuolization and perinuclear hallow (Fig. 1 A). The parotid glands of pilocarpine-radiation group showed a relatively preserved acinar outline and architecture. Some acini showed a mild acinar atrophy with a widen interacinar space and few degenerated acini were also detected while, the nuclei were normal in size and shape and the duct system demonstrated almost intact architecture (Fig. 1 B). The parotid glands of the quercetin-radiation group revealed almost a normal outline and architecture of the acini. Moderate acinar atrophy and degenerative changes were confined to discrete areas in the gland. The acinar cells showed normal- sized and stained nuclei while, the ducts were normal with slight architectural distortion (Fig. 1 C).

\section{Four weeks post-irradiation}

The irradiated parotid glands showed slightly less intense degenerative changes compared to those seen after one week post- irradiation. The acinar vacuolization was markedly reduced, moderate acinar atrophy were seen throughout the gland, while the degeneration of acini was restricted to less and discrete areas. The nuclear pleomorphism and hyperchromatism were persisted to slightly less distributed extent. The ducts showed some cellular vacuolization, while the others demonstrated a loss of normal architecture and degeneration (Fig. 2 A). The pilocarpine-radiation treated parotid glands demonstrated a better integrity of the structural architecture of the gland than after one week post- irradiation. The acinar atrophy was only restricted to few acini, while the acinar degeneration was almost absent, whereas, some acinar cells were still exhibiting nuclear pleomorphism. The ducts showed an intact architecture in the majority of the gland, while scanty ducts showed a structural breakdown. The glands also exhibited a slight periductal inflammatory cell infiltration and intense fibrosis (Fig. 2 B). The glands of the quercetinradiation group demonstrated more or less normal acini with a slight widening of interacinar space while, few acinar cells appeared with nuclear pleomorphism and the ducts showed almost intact architecture (Fig. 2 C).

\section{Sixteen weeks post-irradiation}

Gamma-irradiated glands have relatively restored the normal acinar architecture, but with moderate acinar atrophy and absence of acinar degeneration. The nuclear changes were markedly diminished and manifested as a slight nuclear hyperchromatism. Most of the ducts showed a normal cellular architecture with absent ductal cell vacuolization and degeneration (Fig. 3 A). The parotid glands of pilocarpine-radiation group exhibited tilt toward the normal architecture. The acini demonstrated normal outline and architecture. The nuclei showed normal size, shape and staining. The ducts showed intact architecture and wellarranged ductal cells (Fig. $3 \mathrm{~B}$ ). In the quercetinradiation group, the glands showed relatively preserved acinar outline and architecture. The nuclei of the acinar and ductal cells were uniform in size and stain while, the ducts also have preserved architecture in most areas of the gland with neither vacuolization nor degeneration (Fig. $3 \mathrm{C}$ ).

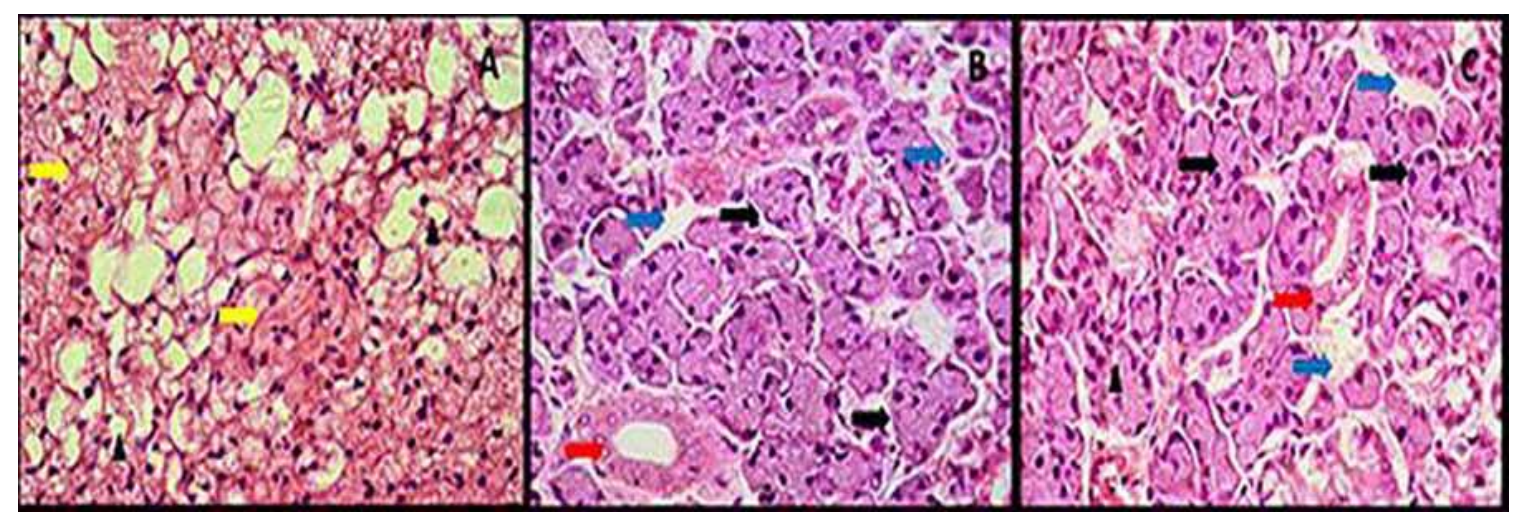

Fig. 1. Photomicrograph of parotid gland one week post-irradiation of radiation group, (A) Pilocarpine-radiation group, (B) Quercetin-radiation group and (C) Showing loss of acinar architecture (yellow arrows). [Acinar vacuolization (arrow heads), normal acinar architecture (black arrows), wide interacinar space (blue arrows) and normal striated duct (red arrows) (H\&E. x400)]. 


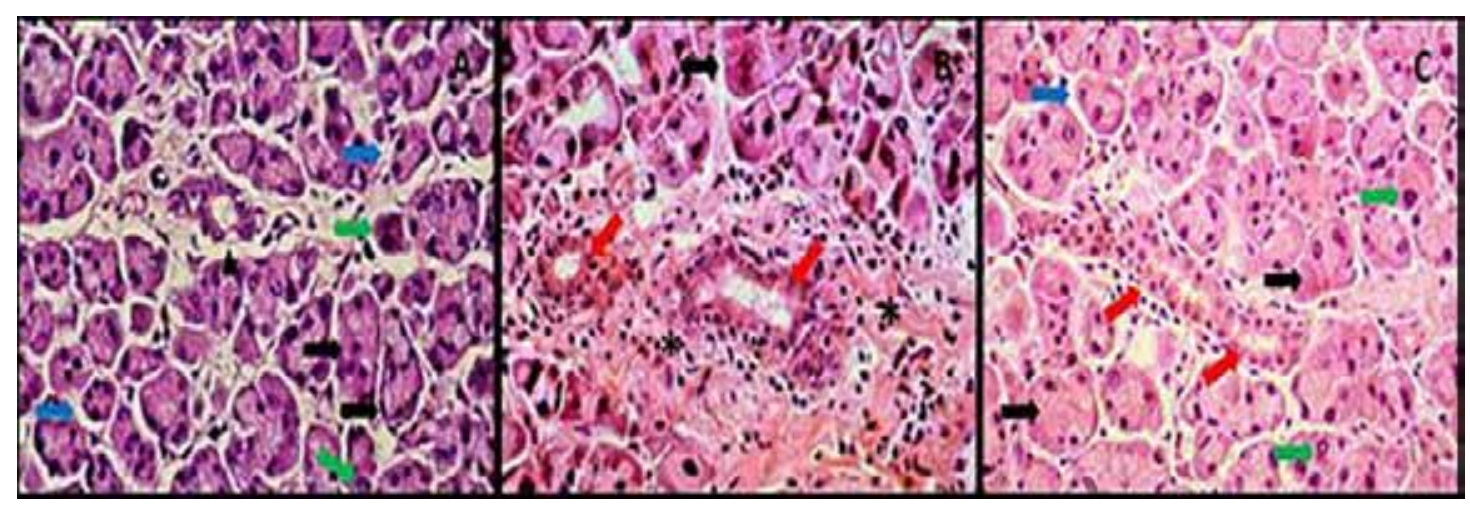

Fig. 2. Photomicrograph of parotid glands four weeks post-irradiation of radiation group, (A) Pilocarpine-radiation group, (B) Quercetin-radiation group, (C) Showing nearly normal acinar architecture. [(black arrows), widen inter acinar space (blue arrows), Vacuolization (arrow heads), nuclear hyperchromatism and pleomorphism (green arrows) and normal striated duct (red arrows). (H\&E. x400)].

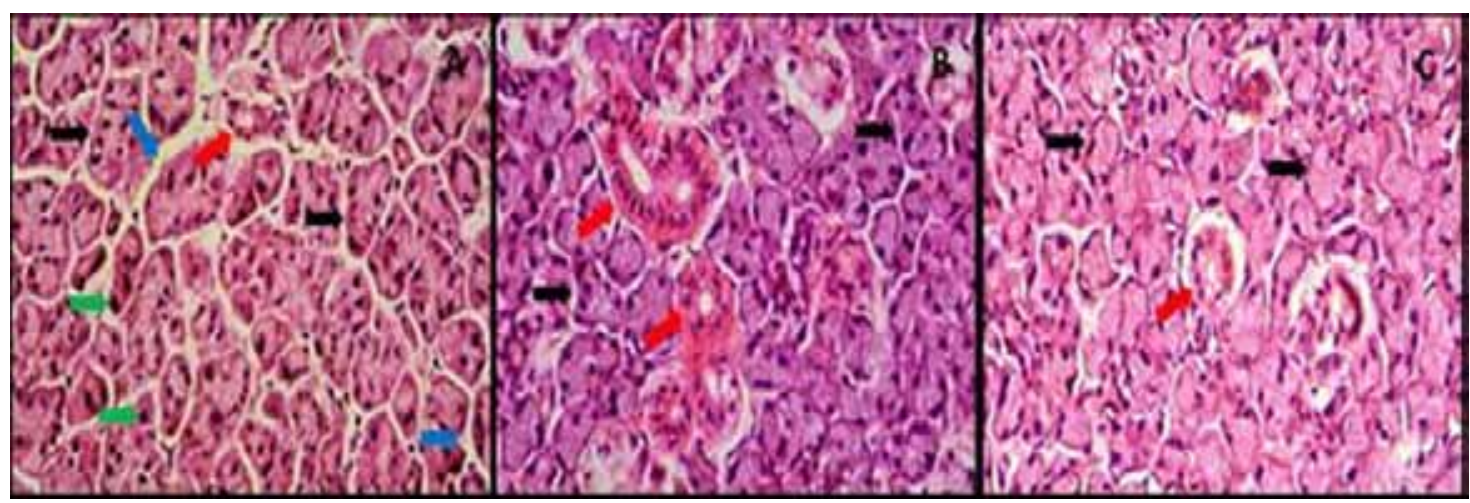

Fig. 3. Photomicrograph of parotid glands sixteen weeks post-irradiation of radiation group, (A) Pilocarpineradiation group, (B) Quercetin-radiation group, (C) Showing normal acinar architecture [(black arrows), widen inter acinar space (blue arrows), nuclear hyperchromatism (green arrows) and normal striated duct (red arrows). (H\&E. x400)].

Immunohistochemical examination

One week post-irradiation

Regarding the radiation group, there were anintense positive immunoreactivity to VEGF expression in the duct, whereas the serous cells showed a moderate to severe reaction. The mean area percent of VEGF expression was $21.38 \pm 3.1$. However, there was a moderate immunoreactivity to VEGF in the ductal and most of the acinar cells of the parotid glands of pilocarpine-radiation group. The mean area percent of VEGF (18.25 \pm 3.3$)$ was significantly lower than those of the radiation group. On the other hand, the glands of the quercetin-radiation group showed similar results to the previous one, where the immunoreactivity to VEGF in both the ductal and acinar cells were moderate. The mean area percent of VEGF expression $((17.01 \pm 4.1)$ showed a significant decrease compared to the radiation group. It was worth noting that no significant difference occurred in respect to VEGF expression between both treatments groups (Fig. 4).

Four weeks post-irradiation

There was an intense immunoreactivity to VEGF in the ducts of the gamma-irradiated glands. Some acini expressed a moderate VEGF reaction while the others were negatively reactive. The mean area percent of VEGF was 17.69 \pm 3.9 . The glands of pilocarpine-radiation group had a mild to moderate VEGF reaction in the ductal cells, while the acinar cells exhibited a negative reaction. The mean area percent of VEGFexpression $(12.91 \pm 2.9)$ was significantly decreased as compared to the radiation group. For the quercetin-radiation group, the whole gland demonstrated a moderate VEGF reactivity. The mean area percent of $\operatorname{VEGF}(14.54 \pm 2.4)$ was significantly reduced compared to the radiation group. Similar to the previous date, no significant difference between treatments groups could be detected (Fig. 4). 


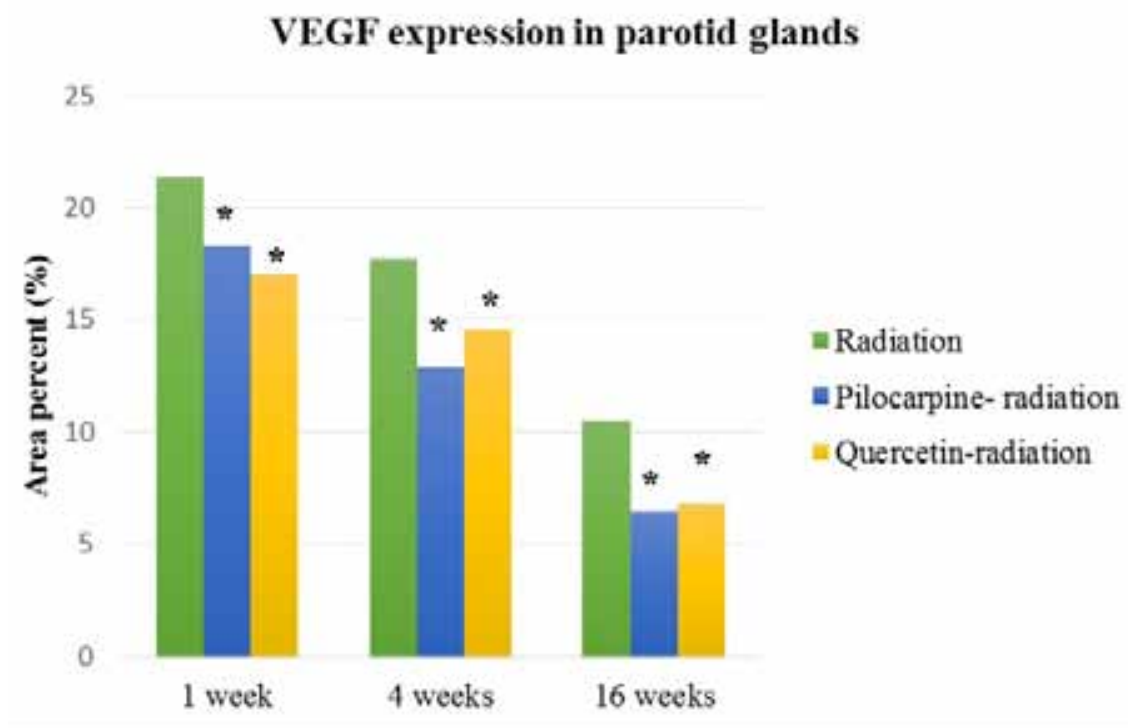

Fig. 4. A histogram representing the mean area percent of VEGF expression in parotid glands of different groups throughout the experiment (* means significant differenceagainst radiation group).

\section{Sixteen weeks post-irradiation}

The whole gland of radiation group showed a mild immunoreactivity to VEGF and the mean area percent of VEGF was $10.54 \pm 4.6$. The glands of the pilocarpine-radiation and quercetinradiation groups revealed a mild VEGF reactivity that was restricted to the ductal cells only while the acinar cells showed a negative reaction. The mean area percentages of VEGF were $6.42 \pm 4.6$ and $6.82 \pm 3.4$, respectively which were significantly decreased compared to the radiation group without a significant difference between both groups (Fig. 4).

\section{Discussion}

Radiation therapy to the head and neck area is one among causes of impaired salivary secretion (Moutsopoulos, 1994). Salivary glands were the most affected organ by radiation in this area however, radiation induced alterations which were variable among the different types of the glands. The present study demonstrated many degenerative changes in the parotid salivary glands when they become exposed to radiation. The cytoplasmic vacuoles detected in the present study in either the acinaror duct cells of parotid glands were comparable to those demonstrated by Sagowski et al. (2004). The observed atrophic changes of serous acini were also documented by Onodera et al. (2006). The acinar atrophic changes had been attributed to radiation-induced apoptosis (Guchelaar et al., 1997), or could be attributed to mast cells activation and release of their secretory products (Henriksson et al., 1994). The detected nuclear pleomorphism and hyperchromatism were also demonstrated in the nuclei of irradiated submandibular glands (Urek et al., 2005).

In the present study, the pilocarpine and quercetin administration before radiation exposure have ameliorated, but did not completely prevent radiation-induced pathological alterations along the experimental periods. The radioprotective effect of pilocarpine and quercetin treatment was manifested by the decreased atrophic changes, acinar cells degeneration, nuclear pleomorphism and hyperchromatism. The relative preservation of the parotid gland architecture due to pilocarpine administration could explain the improved salivary gland function in rats when degranulation was stimulated by pilocarpine administration prior to radiation exposure (Nagler, 2003). Clinically, the administration of pilocarpine during radiation therapy has reduced the development of xerostomia for months even after discontinuation of pilocarpine (Zimmerman et al., 1997).

The improvement of post-radiation xerostomia was more obvious in patients with lower baseline saliva secretion rates (Johnson et al., 1993). In the same way, the quercetin was found to suppress effectively radiation-induced salivary gland impairment as well as to reinforce the normal salivary gland secretion. This could be attributed 
to inhibition of oxidative stress and inflammation associated with radiation exposure, enhancement of calcium uptake and up regulation of aquaporin 5 expression (Takahashi et al., 2015).

In the present study, the irradiated parotid glands showed an intense expression of VEGF. Whereas either the pilocarpine or quercetin administration has reduced the intense expression of VEGF induced by gamma-irradiation exposure. A similar result was obtained by Witte et al. (1989) who found that radiation has induced the release of endothelial cell-derived growth factors. They added that these factors may be involved in the pathogenesis of both the early vascular damage and the late fibrosis which represents a prominent feature of late radiation damage in normal tissues.

\section{Conclusions}

Gamma irradiation has deleterious effects on the histological structure of the parotid salivary glands. Pilocarpine, a parasympathomimetic agonist, has considerably improved the injurious radiation effects on salivary glands. In addition, quercetin, a natural radioprotective agent, has a relative protective effect against radiation induced damage. Therefore, they could be used before radiotherapy schedules to decrease radiation side effects on salivary glands.

\section{Disclosure of Interest}

The authors report no conflicts of interests.

\section{References}

Chambers, M.S., Garden, A.S., Kies, M.S. and Martin, J.W. (2004) Radiation-induced xerostomia in patients with head and neck cancer: Pathogenesis, impact on quality of life, and management. Head \& Neck, 26(9), 796-807.

Chitapanarux, I., Kamnerdsupaphon, P., Tharavichitkul, E., Sumitsawan, Y., Sittitrai, P., Pattarasakulchai, T., Lorvidhaya, Lorvidhaya, Sukthomya, V., Pukanhaphan, N. and Traisatit, P. (2008) Effect of oral pilocarpine on post-irradiation xerostomia in head and neck cancer patients: A single-center, single-blind clinical trial. J. Med. Assoc. Thai. 91(9), 1410-1415.

Comalada, M., Camuesco, D., Sierra, S., Ballester, I., Xaus, J., Gálvez, J. and Zarzuelo, A. (2005) In vivo quercitrin anti-inflammatory effect involves release of quercetin, which inhibits inflammation through down-regulation of the NF- $\mathrm{B}$ pathway. Eur. J. Immunol. 35(2), 584-592.

Coppes, R.P., Vissink, A. and Konings, A.W. (2002) Comparison of radiosensitivity of rat parotid and submandibular glands after different radiation schedules. Radiothe.Oncol. 63(3), 321-328.

Crozier, A., Jaganath, I.B. and Clifford, M.N. (2009) Dietary phenolics: Chemistry, bioavailability and effects on health. Nat.Prod.Rep. 26(8), 1001-1043.

De Groot, H. (1994) Reactive oxygen species in tissue injury. Hepatogastroenterology, 41(4), 328-332.

El-Faramawy, N., Ameen, R., El-Haddad, K. and ElZainy, M. (2013) Effects of gamma radiation on hard dental tissues of albino rats: Investigation by light microscopy. Radiat. Environ. Biophys. 52(3), 375-387.

Ferguson, M.M. (1993) Pilocarpine and other cholinergic drugs in the management of salivary gland dysfunction. Oral Surg. Oral Med. Oral Pathol. Oral Radiol. 75(2), 186-191.

Garg, A.K. and Malo, M. (1997) Manifestations and treatment of xerostomia and associated oral effects secondary to head and neck radiation therapy. $J$. Am. Dent. Assoc., 128(8), 1128-1133.

Guchelaar, H.J., Vermes, A. and Meerwaldt, J.H. (1997) Radiation-induced xerostomia: Pathophysiology, clinical course and supportive treatment. Suppor. Care Cancer, 5(4), 281-288.

Henriksson, R., Fröjd, Ö., Gustafsson, H., Johansson, S., Yi-Qing, C., Franzen, L. and Bjermer, L. (1994) Increase in mast cells and hyaluronic acid correlates to radiation-induced damage and loss of serous acinar cells in salivary glands: The parotid and submandibular glands differ in radiation sensitivity. Br. J. Cancer, 69(2), 320-326.

Johnson, J.T., Ferretti, G.A., Nethery, W.J., Valdez, I.H., Fox, P.C., Ng, D., Muscoplat, C.C. and Gallagher, S.C. (1993) Oral pilocarpine for post-irradiation xerostomia in patients with head and neck cancer. N. Engl. J. Med. 329(6), 390-395.

Looström, H., Åkerman, S., Ericson, D., Tobin, G. and Götrick, B. (2011) Tramadol-induced oral dryness 
and pilocarpine treatment: Effects on total protein and IgA. Arch. Oral Biol. 56(4), 395-400.

Marta, G.N., Silva, V., de Andrade Carvalho, H., de Arruda, F.F., Hanna, S.A., Gadia, R., Silva, J.L. F., Correa, S.F.M., Abreu, C.E.C.V. and Riera, R. (2014) Intensity-modulated radiation therapy for head and neck cancer: Systematic review and metaanalysis. Radiother. Oncol. 110(1), 9-15.

Moutsopoulos, H.M. (1994) Sjöc; gren's Syndrome: Autoimmune Epithelitis. Clin. Immunol. Iimmunopathol. 72(2), 162-165.

Nagler, R.M. (2003) Effects of head and neck radiotherapy on major salivary glands--animal studies and human implications. In Vivo, 17(4), 369-375.

Nishida, S. and Satoh, H. (2009) Possible involvement of $\mathrm{Ca}^{2+}$ Activated $\mathrm{K}^{+}$Channels, SK Channel, in the Quercetin-Induced Vasodilatation. Korean J. Physiol. Pharmacol. 13(5), 361-365.

Okada, D.H., Binder, S.W., Felten, C.L., Strauss, J.S. and Marchevsky, A.M. (1999) "Virtual microscopy" and the internet as telepathology consultation tools: Diagnostic accuracy in evaluating melanocytic skin lesions. Am. J. Dermatopathol. 21(6), 525-531.

Onodera, T., Nasu, M., Iwata, H. and Yosue, T. (2006) Radioprotection of the mice parotid gland by isoproterenol: Study on morphometry of secretory granules and on autoradiography. Oral Radiol. 22(1), 1-6.

Sagowski, C., Wenzel, S., Riehle, I., Tesche, S., Jenicke, L., Zywietz, F., Roeser, K. and Metternich, F.U. (2004) Histomorphologic and salivary gland scintigraphic findings in radiation-induced sialadenitis due to fractionated irradiation of the head and neck region of rats. A model for evaluating potentially radioprotective substances. $H N O, \mathbf{5 2}(1)$, 25-32.

Sumi, M. Tateishi, N., Shibata, H., Ohki, T. and Sata, M. (2013) Quercetinglucosides promote ischemiainduced angiogenesis, but do not promote tumor growth. Life Sci. 93(22), 814-819.

Takahashi, A., Inoue, H., Mishima, K., Ide, F., Nakayama, R., Hasaka, A., Ryo, K., Ito, Y., Sakurai, T., Hasegawa, Y. and Saito, I. (2015) Evaluation of the effects of quercetin on damaged salivary secretion. PLoS One, 10(1), e0116008.

Urek, M.M., Bralic, M., Tomac, J., Borcic, J., Uhac, I., Glazar, I., Antonic, R. and Ferreri, S. (2005) Early and late effects of X-irradiation on submandibular gland: A morphological study in mice. Arch. Med. Res. 36(4), 339-343.

Vivino, F.B. (2001) The treatment of Sjögren's syndrome patients with pilocarpine-tablets. Scand. J. Rheumatol. 30(115), 1-13.

Witte, L., Fuks, Z., Haimovitz-Friedman, A., Vlodavsky, I., Goodman, D.S. and Eldor, A. (1989) Effects of irradiation on the release of growth factors from cultured bovine, porcine, and human endothelial cells. Cancer Res. 49(18), 5066-5072.

Zimmerman, R.P., Mark, R.J., Tran, L.M. and Juillard, G.F. (1997) Concomitant pilocarpine during head and neck irradiation is associated with decreased posttreatmentxerostomia. Int. J. Radiat. Oncol. Biol. Phys. 37(3), 571-575.

(Received 12/8/2018; accepted 4/ 11/2018) 


\title{
تقييم هستولوجى و مناعى لتأثير البيلوكاربين و الكويرسيتين على الغددالنكفية المشععة بأثعةجاما

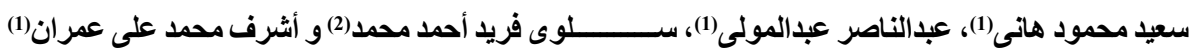

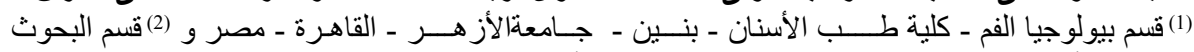 الصحية الأشعاعية ـ المركز القومى لبحوث ولكنولوجيا الأشعاع ـ هيئة الطاقة الذرية ـ القاهرة ـ مصر.
}

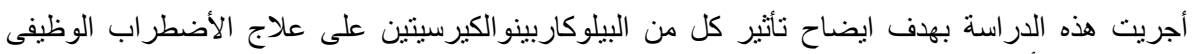

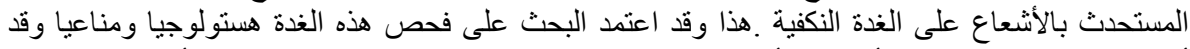

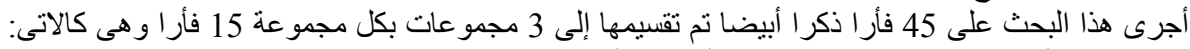

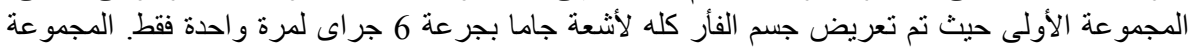

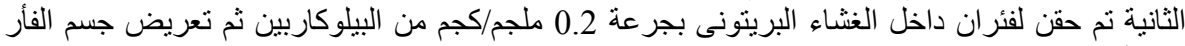

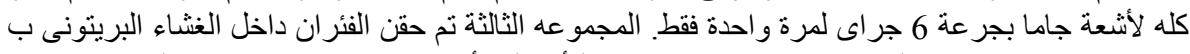

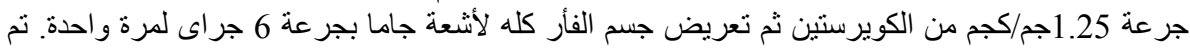

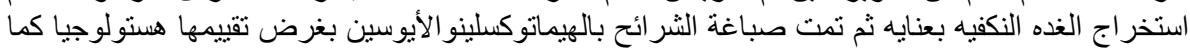

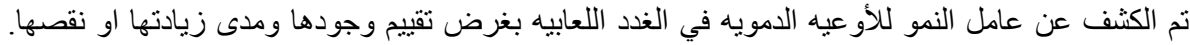

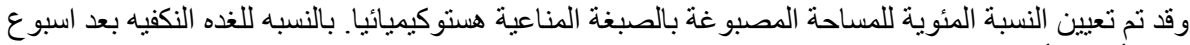

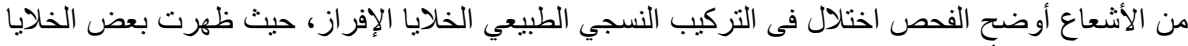

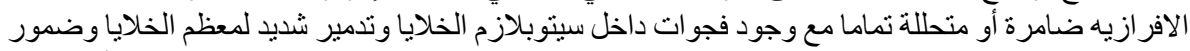

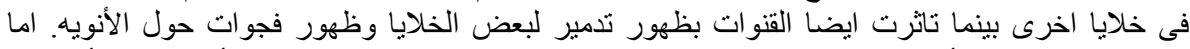

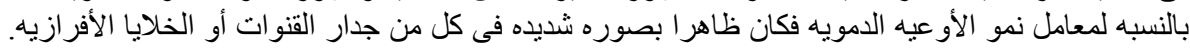

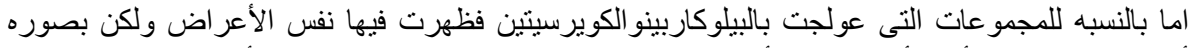

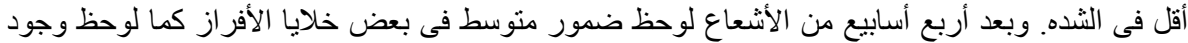

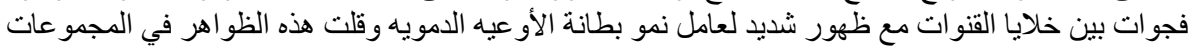

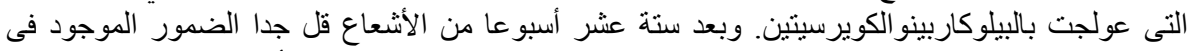

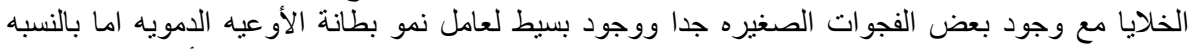

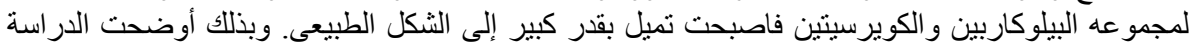

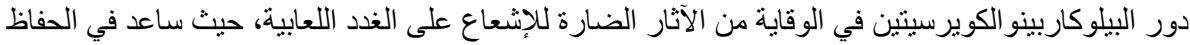
على النمط النسجي الطبيعي ولذلك يوصى بلئ باستعماله قبل التشعيع.
\end{abstract}

